\title{
DOS ESPECIES NUEVAS DE PINGUICULA (LENTIBULARIACEAE) DEL ESTADO DE OAXACA, MEXICO'
}

\author{
SERGIO ZaMUdio y JerZY RZEDOWSKI \\ Instituto de Ecología, Centro Regional del Bajío \\ Apartado Postal 386; 61600 Pátzcuaro, Mich. México
}

\section{RESUMEN}

Se describen como nuevas Pinguicula hemiepiphytica y $P$. utricularioides (Subg. Pinguicula), del estado de Oaxaca, México. Aunque superficialmente semejantes a $P$. moranensis de la sección Orcheosanthus, las dos especies nuevas quedan excluidas de esta sección en virtud del tubo de la corola largamente infundibuliforme. Para ubicar a estos taxa asl como a $P$. crassifolia dentro del subgénero Pinguicula se proponen una nueva sección (Longitubus) y dos subsecciones (Infundibulares y Utriculariopsis).

\begin{abstract}
Pinguicula hemiepiphytica and P. utricularioides (Subg. Pinguicula) from the state of Oaxaca, Mexico, are described as new species. Though similar to $P$. moranensis of section Orcheosanthus, the new species do not fit in this section because of their long funnelform corolla-tube. A section (Longitubus) and two subsections (Infundibulares and Utriculariopsis) are proposed to include these new species together with $P$. crassifolia.
\end{abstract}

Al continuar el estudio de las especies mexicanas del género Pinguicula (Lentibulariaceae), se encontraron especímenes correspondientes a dos taxa del estado de Oaxaca, que por sus características adaptativas y morfológicas se consideran novedades para la ciencia.

La primera planta que se describe en este trabajo ha sido colocada en los herbarios bajo el nombre de $P$. moranensis H.B.K., una especie variable y compleja a la cual con frecuencia se asignan materiales de Pinguicula de corolas grandes y espolones largos que no caben en otra parte. Sin embargo, por el gran tamaño de sus flores y su tendencia a adoptar el hábito epifítico llamó la atención de los autores, quienes consideraron conveniente estudiarla con más detalle.

La revisión de los ejemplares mostró diferencias significativas con respecto a $P$. moranensis, y la observación en el campo confirmó que se trata de una especie no descrita hasta ahora.

\footnotetext{
1 Este trabajo fue realizado con el apoyo del Consejo Nacional de Ciencia y Tecnología, del Centro de Investigación y Desarrollo del Estado de Michoacán y del Consejo de Ciencia y Tecnología del Estado de Querétaro.
} 
Pinguicula hemiepiphytica Zamudio \& Rzedowski sp. nov. (Fig. 1).

Herba perennis, epipetrica vel epiphytica. Folia radicalia dimorpha; "aestivalia" 412, subpetiolata, laminae ellipticae usque ad circulares, margine leviter involutae, (20) 30$70 \mathrm{~mm}$ longae, (12) 20-55 mm latae, petioli 3-15 mm longi; "hiemalia" numerosa, spathulata usque ad oblanceolata, ciliata, 10-20 mm longa, 1-6 mm lata. Pedunculi 1-3, erecti, villosi. Flores $35-80 \mathrm{~mm}$ longi (calcare incluso); calyx bilabiatus, lobis oblongis, 3-6 mm longis, 1.2-3.5 mm latis; corolla bilabiata, violaceo-purpurea vel punicea, fauce alba, labium superum bilobatum, lobis obovato-cuneatis usque ad oblongis, apice rotundatis vel truncatis, 10-19 mm longis, 7-15 mm latis, labium inferum trilobatum, lobis obovato-cuneatis usque ad oblongis, lateralibus apice rotundatis vel truncatis, 8-21 mm longis, 6-17 mm latis, lobus medius lateralibus major, apice emarginatus, $12.5-22 \mathrm{~mm}$ longus, $9.5-22 \mathrm{~mm}$ latus; tubus infundibuliformis, $10-26 \mathrm{~mm}$ longus, 4-7 mm latus, intus dense pubescens, pilis cylindrico-subulatis, multicellularibus, retrorsis, palatum nullum, calcar cylindrico-subulatum, rectum vel recurvatum, 12-30 $\mathrm{mm}$ longum. Capsula subglobosa, 4-6 $\mathrm{mm}$ diametro. Semina numerosa, fusiformia, superficie reticulata, spiculata.

Hierba perenne, epipétrica o epifita, de (6)8 a $20 \mathrm{~cm}$ de alto. Hojas dimórficas, las de "verano" 4 a 12, subpecioladas, lámina eliptica a circular, margen entero, ligeramente involuto, de (20)30 a $70 \mathrm{~mm}$ de largo, por (12)20 a $55 \mathrm{~mm}$ de ancho, peciolo corto, de 3 a $15 \mathrm{~mm}$; hojas de "invierno" numerosas, espatuladas a oblanceoladas, ciliadas, de 10 a $20 \mathrm{~mm}$ de largo por 1 a $6 \mathrm{~mm}$ de ancho. Pedúnculos 1 a 3 por planta, glandular-pilósulos, de 40 a $165 \mathrm{~mm}$ de largo por 1 a $2 \mathrm{~mm}$ de ancho. Flores de 35 a $80 \mathrm{~mm}$ de largo (incluyendo el espolón). Cáliz bilabiado, con pelos glandulares dispersos, el labio superior dividido casi hasta su base en tres lobbulos oblongos de 3.5 a $6 \mathrm{~mm}$ de largo, por (1.5)2 a $3.5 \mathrm{~mm}$ de ancho; el labio inferior dividido hasta $3 / 4$ partes de su longitud en dos lóbulos oblongos, de 3 a $6 \mathrm{~mm}$ de largo, por (1.2)2 a $3 \mathrm{~mm}$ de ancho. Corola bilabiada, violáceo-purpúrea a rosada, con el centro blanco, lóbulos del labio superior obovado-cuneados a oblongos, ápice redondeado a truncado, de 10 a $19 \mathrm{~mm}$ de largo, por 7 a $15 \mathrm{~mm}$ de ancho, lobulos del labio inferior obovado-cuneados a oblongo-cuneados, los laterales con el ápice redondeado a truncado, de 8 a $21 \mathrm{~mm}$ de largo, por 6 a $17 \mathrm{~mm}$ de ancho, el lóbulo medio es más grande, de 12.5 a $22 \mathrm{~mm}$ de largo, por 9.5 a $22 \mathrm{~mm}$ de ancho, con el ápice emarginado; tubo largamente infundibuliforme, de 10 a $26 \mathrm{~mm}$ de largo, por 4 a $7 \mathrm{~mm}$ de ancho, con pelos retrorsos, multicelulares, cilindricos y subulados en su interior; paladar no desarrollado; espolón cilíndrico-subulado, recto o recurvado, de 12 a $30 \mathrm{~mm}$ de largo, por 1 a 2(3) $\mathrm{mm}$ de ancho. Anteras transversalmente elípticas, de $1 \mathrm{~mm}$ de largo, los filamentos glandulosos. Estigma bilobado, el labio inferior agrandado, hemicircular, fimbriado, de $3 \mathrm{~mm}$ de diámetro. Cápsula subglobosa, de 4 a $6 \mathrm{~mm}$ de diámetro, glandularpubescente. Semillas numerosas, fusiformes, con la superficie reticulada y espiculada.

TIPO: México, Oaxaca, $58 \mathrm{~km}$ al $\mathrm{N}$ de Ixtlán, por la carretera a Valle Nacional, sobre taludes, entre colonias de musgos, bosque mesófilo de montaña, alt. $2200 \mathrm{~m}, 12 . X .1987$, S. Zamudio 5630 (HOLOTIPO: IEB. ISOTIPOS: ENCB, CHAPA, MEXU). 
$A^{\prime}$

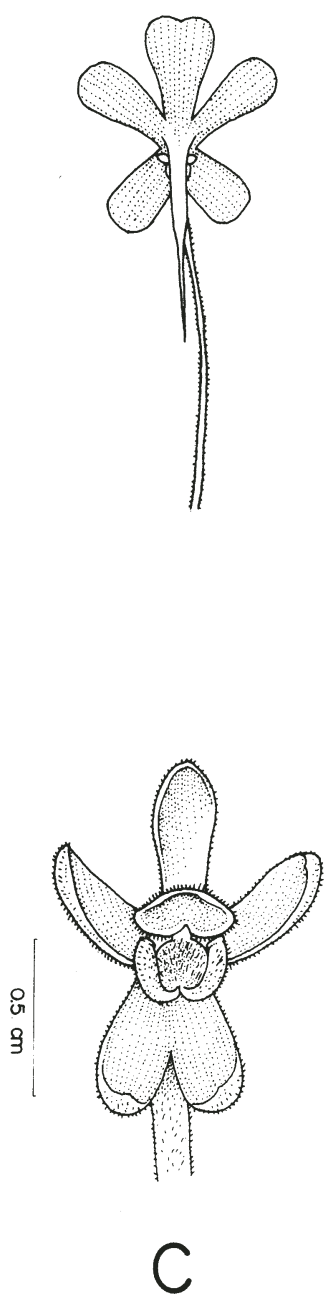

A
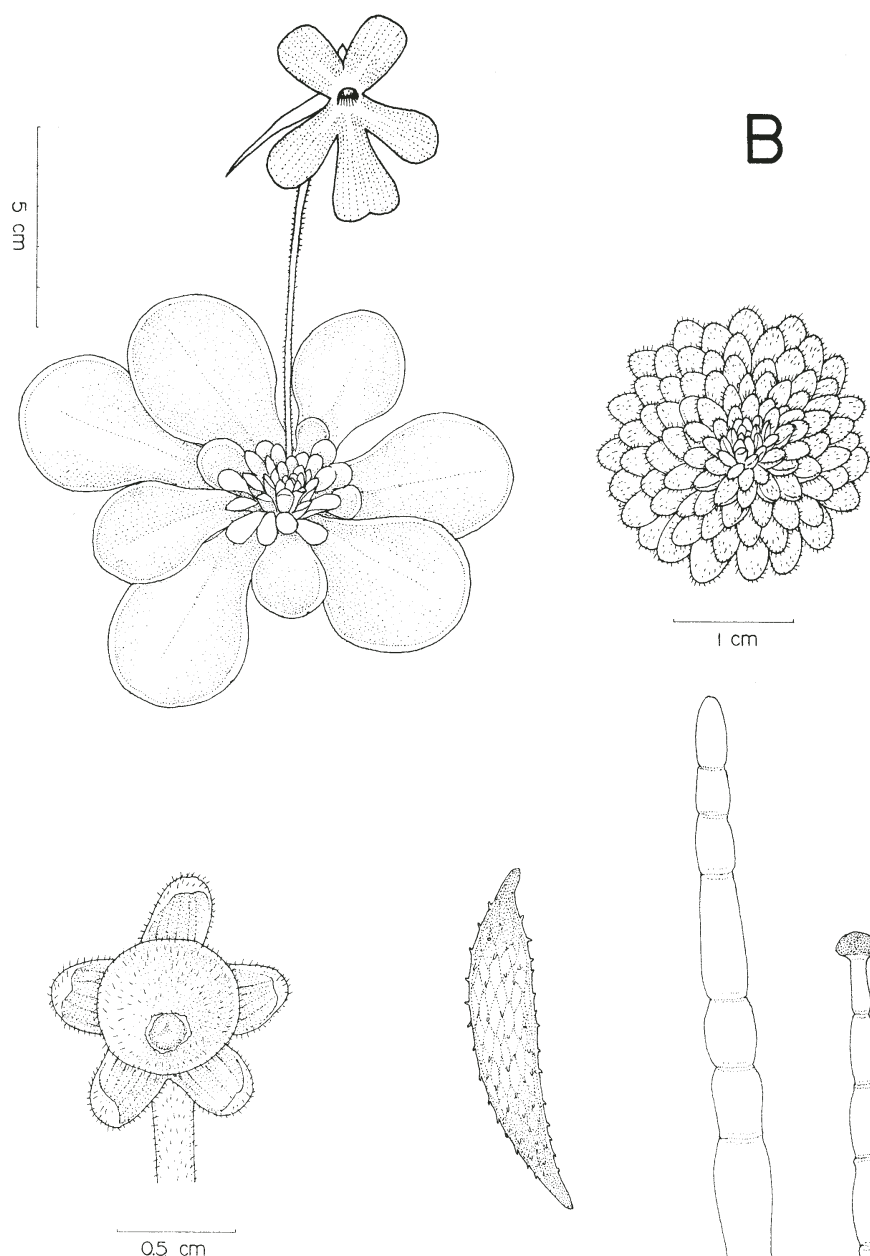

D

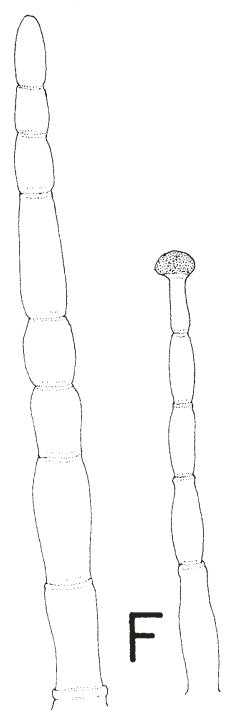

Fig. 1. Pinguicula hemiepiphytica. A. Hábito, planta con roseta de "verano"; A'. Vista posterior de la flor mostrando el tubo largamente infundibuliforme; B. Roseta de "invierno"; C. Cáliz mostrando los estambres y el ovario; D. Fruto; E. Semilla; F. Pelos del interior del tubo de la corola. Dibujo de Miguel Carmona. 
Material adicional examinado: MEXICO, Oaxaca, Along rd. betw. Ixtlan and Valle Nacional, 32 mi. by rd. above Valle Nacional, ca. $17^{\circ} 35^{\prime} \mathrm{N}, 96^{\circ} 25^{\prime} \mathrm{W}$; on steep mossy banks, alt. 7000 ft., fls. lavender-pink, 20.VI.1969, G.L. Webster \& G.J. Breckon 15391 (MEXU); $60 \mathrm{~km}$ al $\mathrm{N}$ de Ixtlán, sobre la carretera a Tuxtepec, bosque mesófilo de montaña, alt. $2150 \mathrm{~m}$, planta epifita, flores moradas, 26.V.1973, Rzedowski 30616 (ENCB, CHAPA, MEXU); $46 \mathrm{~km}$ al SSW de Valle Nacional, sobre la carretera a Oaxaca, alt. $2050 \mathrm{~m}$, planta epifita, flores rosadas, 26.VI.1975, Rzedowski 33357 (ENCB); Hwy 175, between Oaxaca and Tuxtepec, steep slopes on the Gulf side where extra rainfall is common, in the cloud forest, alt. 6600 ft., 15.VIII.1975, Torke, Dunn \& LeDoux 510 (ENCB, INIF, MEXU); 10 $\mathrm{km}$ al S de La Esperanza, carretera Oaxaca-Tuxtepec, Distrito de Tuxtepec, bosque mesófilo de montaña de Engelhardtia mexicana, alt. $2030 \mathrm{~m}$, hierba con flores de color lila, abundante en los declives de la carretera, 3.VIII.1981, Abisal Garcia M. et al. 667 (IEB, MEXU); La Cascada de Comaltepec, brecha 64, bosque mixto de Weinmannia y pino, planta insectivora, flores rosas, 16.IV.1982, $R$. Cedillo T. con R. Torres y D. Lorence 1226 (MEXU); $5 \mathrm{~km}$ al $\mathrm{N}$ de la desviación a San Pedro Yolox, bosque de pino-encino, alt. 2290 $\mathrm{m}$, planta epifita, sobre encinos, flor color lila,1.VII.1982, $R$. Cedillo Trigos y $R$. Torres 1581 (MEXU); $5 \mathrm{~km}$ al $\mathbf{N}$ de Cerro Humo Chico, carr. Ixtlán a Valle Nacional, bosque de encinos, flores lilas, epifitas sobre encino, 26.IX.1982, R. Cedillo T. y R. Torres 1866 (MEXU); 14 $\mathrm{km}$ al S de La Esperanza, carretera Tuxtepec-Oaxaca, municipio de Comaltepec, Distrito de Ixtlán, bosque mesofilo, alt. $1900 \mathrm{~m}$, hierba rupicola, flores color lila, 26.VIII.1986, $R$. Torres y L. Cortés 8685 (IEB,MEXU); Depto. de Ixtlán, 22 km al W de La Esperanza, carr. Tuxtepec-Oaxaca, mpio. Comaltepec, bosque mesofilo, alt. $1900 \mathrm{~m}, 9 . \mathrm{IV} .1987, R$. Torres C., A. García y L. Cortés 9537 (MEXU).

Esta especie ha sido señalada con frecuencia como epifita por los colectores, aunque crece también sobre rocas y taludes en lugares muy húmedos y asociada con colonias de musgos; se ha encontrado entre 1900 y $2300 \mathrm{~m}$ de altitud, en el límite superior del bosque mesófilo de montaña de Engelhardtia mexicana Standley y Quercus spp.

Florece de mayo a septiembre, cuando presenta la "roseta de verano".

Hasta ahora sólo se conoce de la vertiente septentrional de la Sierra de Juárez en el estado de Oaxaca, sin embargo se podría esperar su presencia en los estados vecinos, tomando en cuenta que el bosque de Engelhardtia mexicana también se conoce de Veracruz y posiblemente existe en Chiapas (Rzedowski y Palacios-Chávez, 1977).

Es notable la tendencia de la especie a adoptar el hábitat epifítico, ya que este comportamiento sólo se conocla con anterioridad para el género en el caso de $P$. lignicola Barnh. de Cuba y F. casabitoana Jiménez de Santo Domingo (Casper, 1987), especies no directamente emparentadas con $P$. hemiepiphytica.

Por consiguiente, la tendencia hacia el epifitismo en $P$. hemiepiphytica parece ser el resultado de convergencia evolutiva dentro del género; este es un fenómeno poco estudiado hasta ahora, pero su aparición en regiones con clima tropical o subtropical sugieren la incursión hacia nuevos hábitats de un género típicamente terrestre.

Pinguicula hemiepiphytica se ubica en el subgénero Pinguicula (Casper, 1962), dentro del cual muestra afinidad con las especies de la sección Orcheosanthus A. DC., 
pero formalmente queda excluida de ahi por el tubo de la corola largamente infundibuliforme, ya que según la diagnosis de esta sección el tubo es cortísimo, ampliamente infundibuliforme y notoriamente más corto que los lóbulos de la corola y el espolón. Tal hecho coloca a $P$. hemiepiphytica en la misma situación de $P$. crassifolia Zamudio (Zamudio, 1988). La ubicación definitiva de estas especies dentro del subgénero será discutida con mayor amplitud más adelante.

Además de sus relaciones con $P$. crassifolia por la forma y longitud del tubo de la corola, $P$. hemiepiphytica se parece mucho a $P$. moranensis, con la que ha sido confundida por muchos años. Los individuos pertenecientes a estas dos especies se pueden diferenciar con facilidad por su apariencia general en fresco; por desgracia, no sucede lo mismo después de haberse secado, por lo que resulta difícil su identificación en ejemplares de herbario, sobre todo cuando están mal prensados.

Las diferencias más notorias que podemos señalar entre este grupo de especies se resumen en el Cuadro 1.

Cuadro 1. Expresa las diferencias que existen en algunas estructuras y el comportamiento fenológico y ecológico entre Pinguicula moranensis, $P$. crassifolia, $P$. hemiepiphytica y $P$. utricularioides.

\begin{tabular}{|c|c|c|c|c|}
\hline & P. moranensis & P. crassifolia & P. hemiepiphytica & P. utricularioides \\
\hline $\begin{array}{l}\text { Largo de la flor } \\
\text { Tubo de la corola: }\end{array}$ & $30-64 \mathrm{~mm}$ & $25-30 \mathrm{~mm}$ & $35-80 \mathrm{~mm}$ & $27-37 \mathrm{~mm}$ \\
\hline $\begin{array}{l}\text { Largo } \\
\text { Forma }\end{array}$ & $\begin{array}{l}3-6 \mathrm{~mm} \\
\text { cortamente } \\
\text { infundibuliforme }\end{array}$ & $\begin{array}{l}\text { 8-15 mm } \\
\text { largamente } \\
\text { infundibuliforme }\end{array}$ & $\begin{array}{l}10-26 \mathrm{~mm} \\
\text { largamente } \\
\text { infundibuliforme }\end{array}$ & $\begin{array}{l}17-23 \mathrm{~mm} \\
\text { largamente } \\
\text { infundibuliforme }\end{array}$ \\
\hline Largo del espolón & $14-44 \mathrm{~mm}$ & $8-25 \mathrm{~mm}$ & $12-30 \mathrm{~mm}$ & $5-8 \mathrm{~mm}$ \\
\hline Floración & enero-diciembre & marzo-junio & mayo-septiembre & marzo-? \\
\hline $\begin{array}{l}\text { Roseta presente en } \\
\text { la floración }\end{array}$ & de verano & de invierno & de verano & de invierno \\
\hline Hábito & terrestre & epipétrica & epifita o epipétrica & $?$ \\
\hline $\begin{array}{l}\text { Amplitud altitudinal } \\
\text { conocida }\end{array}$ & $1150-2850 \mathrm{~m}$ & $2850-3000 \mathrm{~m}$ & $1900-2300 \mathrm{~m}$ & $1800-2000 \mathrm{~m}$ \\
\hline
\end{tabular}

La segunda especie tratada en este trabajo sólo se ha colectado en la parte alta del Cerro Azul, ubicado al norte de Niltepec en el estado de Oaxaca; los especímenes muestran flores con rosetas de invierno y no se conoce la roseta de verano. Al parecer es una planta de distribución muy restringida con características morfologicas únicas que la separan con facilidad de las demás especies mexicanas.

Dada su importancia para el esclarecimiento de algunos problemas taxonómicos en el grupo al que pertenece, se ha decidido darla a conocer, aunque no se cuenta con toda la información que sería deseable para una descripción completa. 
Pinguicula utricularioldes Zamudio \& Rzedowski sp. nov. (Fig. 2).

Herba perennis. Folia radicalia dimorpha; interiora (probabiliter "hiemalia") 14-23, spathulata, 5-20 mm longa, 1-2.5 mm lata; exteriora ("aestivalia"?) longe petiolata, laminae induplicatae, sacciformes, 2-4 mm diametro, petioli lineares, $10-25 \mathrm{~mm}$ longi. Pedunculi erecti, villosi. Flores $27-37 \mathrm{~mm}$ longi (calcare incluso); calyx bilabiatus, labium superum trilobatum, lobis oblongis, 2-3 mm longis, 1-2 mm latis, labium inferum indivisum, oblongum, 2-3.5 mm longum, 2-2.5 mm latum, apice truncatum vel emarginatum; corolla bilabiata, rubra (rubro-scarlatina fide MacDougall), labium superum bilobatum, lobis obovato-cuneatis, apice emarginatis, $4.5-10 \mathrm{~mm}$ longis, 4-7 mm latis; labium inferum quam supero majus, trilobatum, lobis obovatis usque ad ellipticis, $8-11 \mathrm{~mm}$ longis, $5-10 \mathrm{~mm}$ latis, tubus infundibuliformis, 17-23 mm longus, 5-10 mm latus, intus dense pubescens, pilis subulatis, multicellularibus, retrorsis, palatum nullum; calcar subulatum, brevissimum, 5-8 mm longum. Ovarium subglobosum. Capsula ignota. Semina ignota.

Hierba perenne, de hasta $11 \mathrm{~cm}$ de altura (incluyendo la inflorescencia). Hojas basales dimórficas, las interiores, (probablemente "de invierno") 14 a 23, espatuladas, de 5 a $20 \mathrm{~mm}$ de largo, por 1 a $2.5 \mathrm{~mm}$ de ancho, vilosas en el haz, con pelos glandulares multicelulares; las hojas exteriores escasas, 1 a 4 , (estas posiblemente correspondan a las hojas de la roseta de "verano"), largamente pecioladas, lámina induplicada, formando una especie de saco subgloboso, de 2 a $4 \mathrm{~mm}$ de diámetro, peciolo linear, de 10 a 25 $\mathrm{mm}$ de largo, glandular-viloso. Pedúnculo linear, de 5 a $9 \mathrm{~cm}$ de largo, por $1 \mathrm{~mm}$ de ancho, viloso. Flores de 27 a $37 \mathrm{~mm}$ de largo (incluyendo el espolón). Cáliz bilabiado, glandular -viloso, el labio superior dividido hasta $3 / 4$ partes de su longitud en tres lóbulos oblongos, de 2 a $3 \mathrm{~mm}$ de largo por 1 a $2 \mathrm{~mm}$ de ancho; labio inferior no dividido, oblongo, de 2 a $3.5 \mathrm{~mm}$ de largo, por 2 a $2.5 \mathrm{~mm}$ de ancho, ápice truncado o emarginado. Corola bilabiada, roja (rojo escarlata según MacDougall), labio superior dividido en dos lóbulos, obovado-cuneados, de 4.5 a $10 \mathrm{~mm}$ de largo, por 4 a $7 \mathrm{~mm}$ de ancho, ápice emarginado; labio inferior más grande que el superior, dividido en tres lóbulos obovados a elípticos, de 8 a $11 \mathrm{~mm}$ de largo, por 5 a $10 \mathrm{~mm}$ de ancho; tubo largamente infundibuliforme, de 17 a $23 \mathrm{~mm}$ de largo, por 5 a $10 \mathrm{~mm}$ de ancho, glabrescente en la parte externa, con abundantes pelos glandulares multicelulares, subulados y retrorsos en la garganta; paladar no desarrollado; espolón corto, subulado, de 5 a $8 \mathrm{~mm}$ de largo, glandular-viloso por fuera, con pelos glandulares subulados y retrorsos por dentro. Estambres dos, ligeramente geniculados, de 2.2 a $2.8 \mathrm{~mm}$ de largo, por 0.3 a $0.5 \mathrm{~mm}$ de ancho, anteras glabras. Ovario subgloboso, glandular-pubescente, lóbulo inferior del estigma agrandado, hemicircular, fimbriado, de $\pm 2 \mathrm{~mm}$ de alto por 2.5 a $2.8 \mathrm{~mm}$ de ancho, papiloso en la cara interna, viloso en la cara externa. Fruto desconocido. Semilla desconocida.

TIPO: México, Oaxaca, Cerro Azul al norte de Niltepec, alt. 6000-7000 ft., 7-III1956, T. MacDougall s.n. (HOLOTIPO: MEXU, ISOTIPO: IEB).

Esta especie hasta ahora sólo se conoce de la colecta de MacDougall, los datos de campo son escuetos y no se describe con precisión el hábitat que ocupa. 


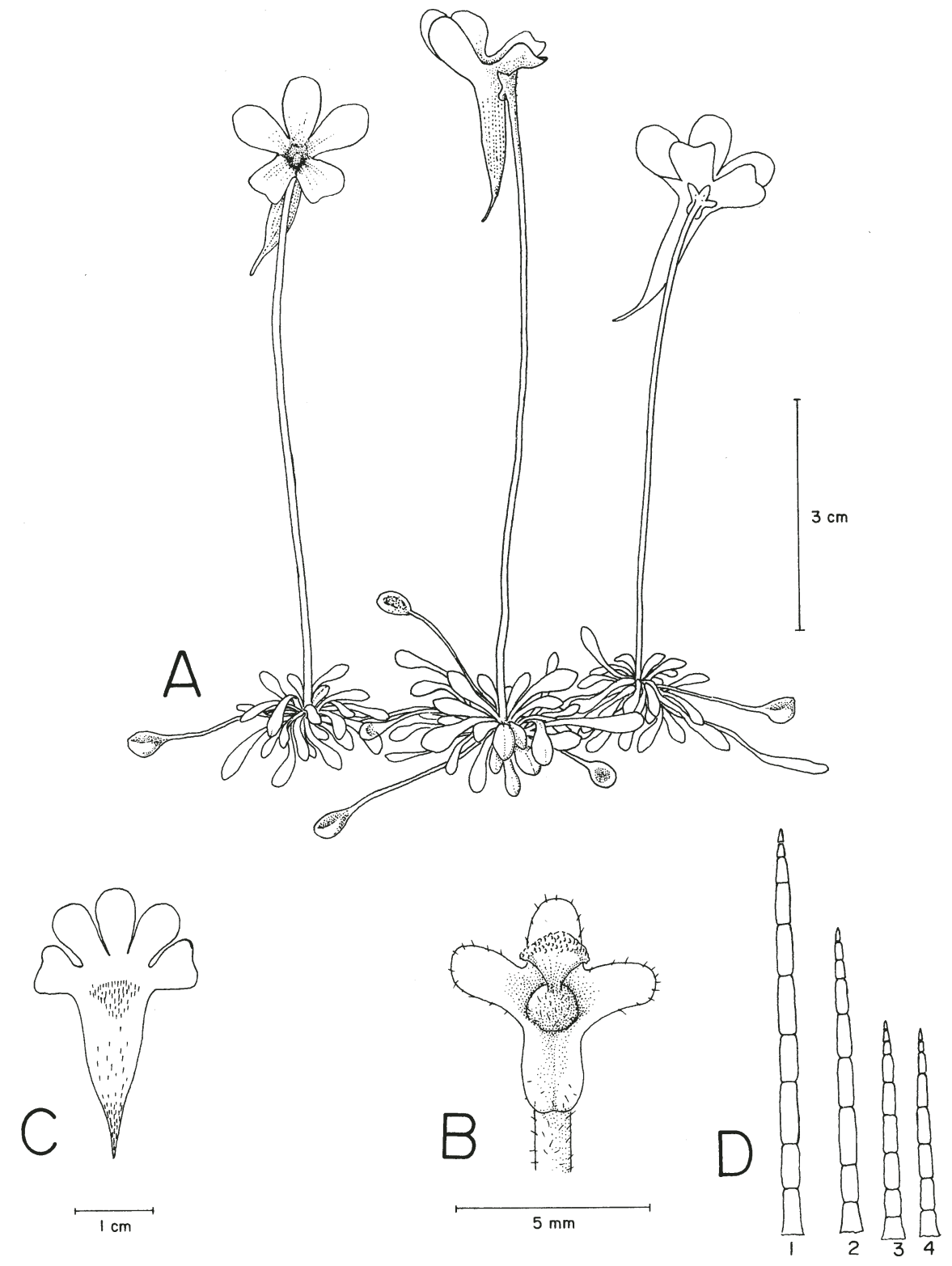

Fig. 2. Pinguicula utricularioides. A. Hábito, planta con roseta de "invierno" y hojas largamente pecioladas con lámina en forma de saco (probablemente de "verano"); B. Cáliz mostrando el ovario; C. Corola abierta mostrando los pelos de la garganta, tubo y espolón; D. Pelos del interior de la corola: 1 y 2 de la garganta y tubo de la corola, 3 y 4 del espolón. Dibujo de Rodrigo Tavera. 
$P$. utricularioides por sus características adaptativas se distingue de todas las especies del género, destacando entre ellas la fuerte modificación de la lámina en las hojas externas (que no sabemos con certeza si corresponden a las hojas de la roseta de "verano"), en las que el margen de la hoja se enrolla hacia adentro hasta formar una cavidad subglobosa, que aparentemente funciona como una trampa para pequeños insectos y otros invertebrados. Si tal función se confirma, la mencionada modificación adaptativa podría significar una relación estrecha con el género Utricularia.

Tal estructura, no conocida con anterioridad para el género Pinguicula, resulta sumamente novedosa y en este momento no podemos valorar su significado evolutivo, ya que no se han visto especímenes vivos. Estudiar su función específica y aclarar si realmente corresponden a las hojas de la roseta de "verano" son problemas que quedan por resolver en el futuro.

Resalta también el hecho de que el labio inferior del cáliz no está dividido y sólo en algunos casos el ápice se aprecia ligeramente emarginado, por lo que el cáliz se observa en su conjunto con sólo cuatro lóbulos. Esta característica se conocía con anterioridad en Pinguicula lignicola y $P$. cladophila Ernst, distribuidas en las Antillas, así como en $P$. crystallina Sibth. ex Sibth. \& Smith y $P$. hirtiflora Tenore del sur de Europa, todas ellas pertenecientes al subgénero Isoloba, sensu Casper (1966).

El tubo de la corola largamente infundibuliforme, con un espolón cortísimo, casi vestigial, relaciona a $P$. utricularioides con $P$. crassifolia y $P$. hemiepiphytica, las que en conjunto forman un grupo particular dentro del subgénero Pinguicula que por sus características florales no se puede ubicar satisfactoriamente en las secciones descritas hasta ahora.

Las especies en cuestión presentan el tubo de la corola largamente infundibuliforme pero variable en longitud y en su relación con los lóbulos y el espolón. En $P$. crassifolia el tubo es largo, pero no supera la longitud de los lóbulos y el espolón; en $P$. hemiepiphytica el tubo es subigual o mayor que los lóbulos de la corola y el espolón y en $P$. utricularioides el tubo es notoriamente más largo que los lóbulos de la corola y el espolón. Otras diferencias entre los mencionados taxa se aprecian en el Cuadro 1.

La afinidad más cercana de este conjunto de especies se encuentra con la sección Orcheosanthus y en menor grado con la sección Pinguicula. De la primera se distinguen por los lóbulos de la corola en ocasiones emarginados, el tubo largamente infundibuliforme, subigual o mayor que los lóbulos y el espolón; de la sección Pinguicula se separan por las hojas dimórficas, la ausencia de hibernáculos, la corola grande, con algunos lóbulos emarginados, el tubo de la corola largamente infundibuliforme y el espolón incurvado.

La existencia de este grupo de especies con el tubo de la corola excepcionalmente largo plantea la necesidad de proponer una nueva sección así como dos subsecciones dentro del subgénero Pinguicula para darles cabida, como se hace a continuación.

Sect. Longitubus Zamudio \& Rzedowski sect. nov.

Folia biformia hibernaculis nullis, corolla ringens, magna, profunde bilabiata, labio infero quam supero majore lobis subaequalibus integerrimis vel emarginatis; tubus longe infundibuliformis; calcar incurvatum tubum subaequans vel minor. 
Hojas dimorfas, hibernáculo ausente, corola grande, profundamente bilabiada, roja - rojo-violácea, labio inferior más grande que el superior, sus lóbulos subiguales, enteros o emarginados, tubo largamente infundibuliforme, espolón incurvado, subigual o más corto que el tubo.

Tipo: Pinguicula crassifolia Zamudio.

Subsect. Infundibulares Zamudio \& Rzedowski subsect. nov.

Folia "hiemis" crassa lanceolata, oblongo-spathulata usque ad anguste obtrullata, non in acumen attenuata; folia "aestatis" elliptica, ovata vel suborbiculata, solum plus minusve adpressa.

Hojas de "invierno" carnosas, lanceoladas, oblongo-espatuladas a obtruladas en contorno, no acuminadas; hojas de "verano" elípticas, ovadas o suborbiculadas, más o menos adpresas al suelo.

Tipo: Pinguicula crassifolia Zamudio.

Otra especie contenida en esta subsección es $P$. hemiepiphytica Zamudio \& Rzedowski, descrita en este mismo trabajo.

Subsect. Utriculariopsis Zamudio \& Rzedowski subsect. nov.

Folia interiora spathulata, ciliolata, non in acumen attenuata, folia exteriora longe petiolata, lamina induplicata sacciformis.

Hojas interiores espatuladas, cilioladas, no acuminadas; hojas exteriores largamente pecioladas, lámina induplicada, en forma de saco.

Tipo: Pinguicula utricularioides Zamudio \& Rzedowski.

\section{AGRADECIMIENTOS}

Se agradece a los encargados de los herbarios ENCB, INIF y MEXU por el préstamo de ejemplares botánicos.

\section{LITERATURA CITADA}

Casper, S. J. 1962. Revision der Gattung Pinguicula in Eurasien. Repert. Spec. Nov. 66 (1/2): 1-148.

Casper, S. J. 1966. Monographie der Gattung Pinguicula L. Bibliotheca Botanica 31(127/128): 1-209.

Casper, S. J. 1987. On Pinguicula lignicola, an epiphytic heterophyllic member of the Lentibulariaceae in Cuba. PI. Syst. Evol. 155: 349-354.

Rzedowski, J. y R. Palacios-Chávez. 1977. El bosque de Engelhardtia (Oreomunnea) mexicana en la región de la Chinantla (Oaxaca, México). Una reliquia del Cenozoico. Bol. Soc. Bot. Méx. 36: 93123.

Zamudio, S. 1988. Dos nuevas especies de Pinguicula (Lentibulariaceae) del centro y norte de México. Acta Bot. Mex. 3: 21-28. 
NOTA: Cuando esta publicación estaba a punto de salir a la luz, llegó a nuestras manos el siguiente articulo: Speta, F. \& F. Fuchs. 1989. Drei neue PinguiculaArten der Sektion Orcheosanthus DC. aus Mexico. Phyton (Austria) 29(1): 93-103, en donde se describe, entre otras, Pinguicula laveana Speta \& Fuchs, a partir de material colectado en la Sierra Mixe, Oaxaca por A. Lau (No. 009), y cultivado en el Jardín Botánico de Linz.

Es posible que $P$. laueana y $P$. hemiepiphytica sean conespecificas, pero la descripción de la primera no es suficientemente detallada y faltan datos precisos de localidad y habitat para poder tomar una decisión definitiva. Sería recomendable comparar material vivo de ambas entidades para aclarar satisfactoriamente esta duda. 